\title{
Determining the temporal interaction of Camellia cultivar flowering periods and camellia petal blight disease presence in Auckland, New Zealand
}

\author{
Emma Bodley' \& Rebecca Stanley²
}

\begin{abstract}
Botanic gardens hold diverse and valuable living collections that provide a number of research, conservation and education opportunities. Auckland Botanic Gardens (ABG) has an internationally significant collection of species and cultivar camellias that have been used for scientific research into understanding which species are susceptible to camellia petal blight. ABG has surveyed all cultivars and species in its collection over multiple years to identify which camellias are affected by petal blight and which avoid it, based on their flowering period. A total of 363 camellias were assessed for petal blight over 6 years of surveying. It was found that 245 cultivars and 6 species were susceptible to the disease, while 68 cultivars and 32 species did not display any visual symptoms. These are important findings, as enthusiasts and breeders alike may use them to inform their respective interests.
\end{abstract}

\section{Introduction}

Botanic gardens play a key role in supporting and facilitating research. They hold diverse and important plant collections that provide researchers with convenient access to plants which are well documented on databases. Horticultural and botanical experts are available to make daily observations on growth, maintenance, and pests and diseases. Information that staff acquire through regular observations is captured on the databases; this provides an important life history of the plants, revealing information about them over time, such as their susceptibility to pests or diseases.

Camellias have been grown in New Zealand as a common garden plant for decades. They provide autumn and winter interest in gardens as specimen trees and are also used as hedging (Fig. 1). They attract insect pollinators and birds to gardens at a time of year when there are fewer flowers around for foraging.

Since the introduction to New Zealand in the early 1990s of the fungus Ciborinia camelliae, the causal agent of camellia petal blight, many Camellia cultivars have no longer been useful amenity plants because this disease infects the blooms, causing them to turn brown and fall early (Fig. 2). The fungus can be confirmed by removing a flower from the plant and looking on the back side for a grey ring of fungal hyphae at the base of the petals (Fig. 3). C. camelliae only infects the floral parts of a plant and is not known to infect any other genera

${ }^{1}$ Emma Bodley is Botanical Records and Conservation Specialist at Auckland Botanic Gardens.

Address: 102 Hill Road, Manurewa, Auckland, New Zealand.

Email: emma.bodley@aucklandcouncil.govt.nz

${ }^{2}$ Rebecca Stanley is a Curator at Auckland Botanic Gardens.

Address: as above. 
2 Emma Bodley \& Rebecca Stanley

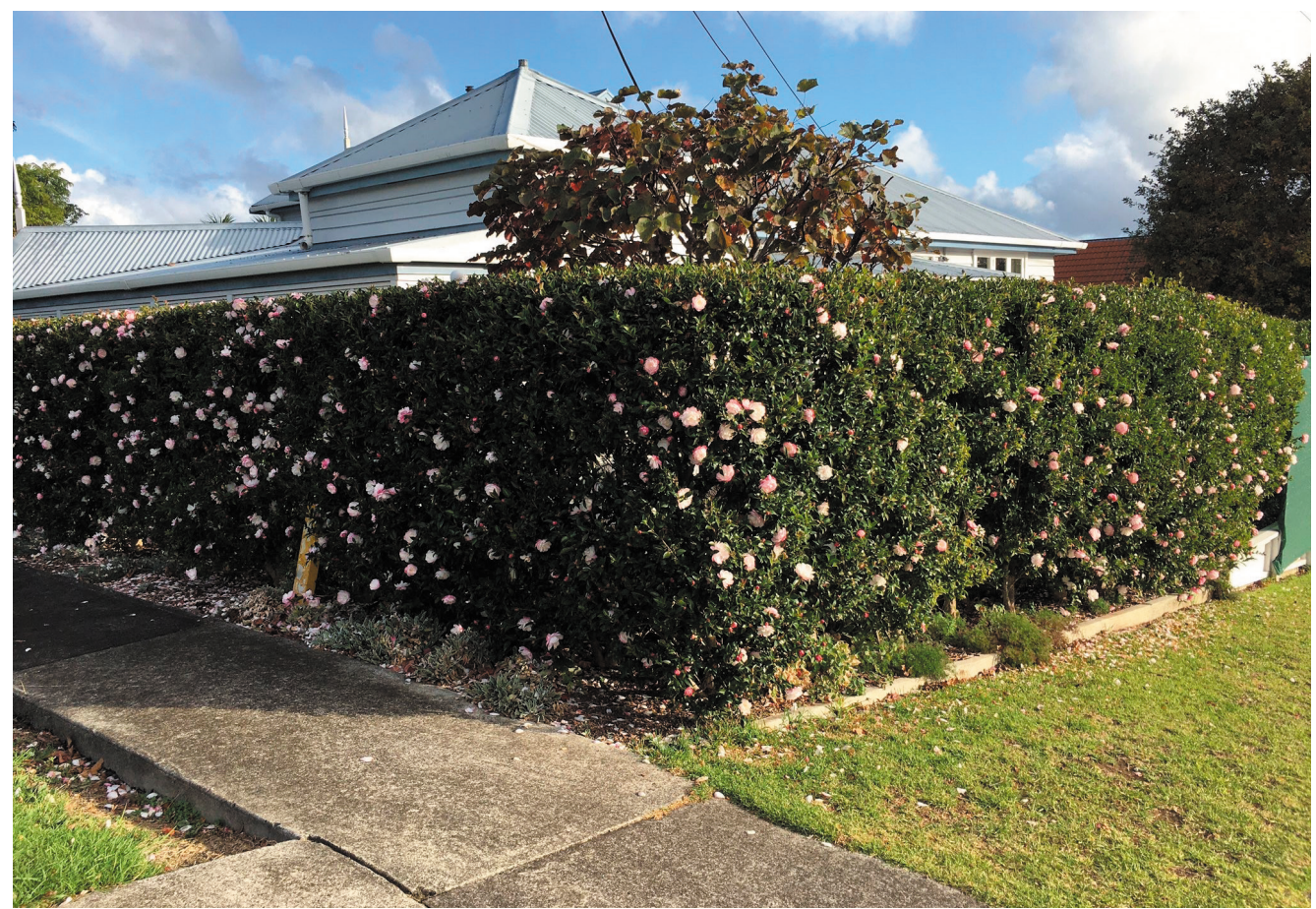

Fig. 1 Camellias are commonly used as hedging in New Zealand. Photo: Rebecca Stanley.

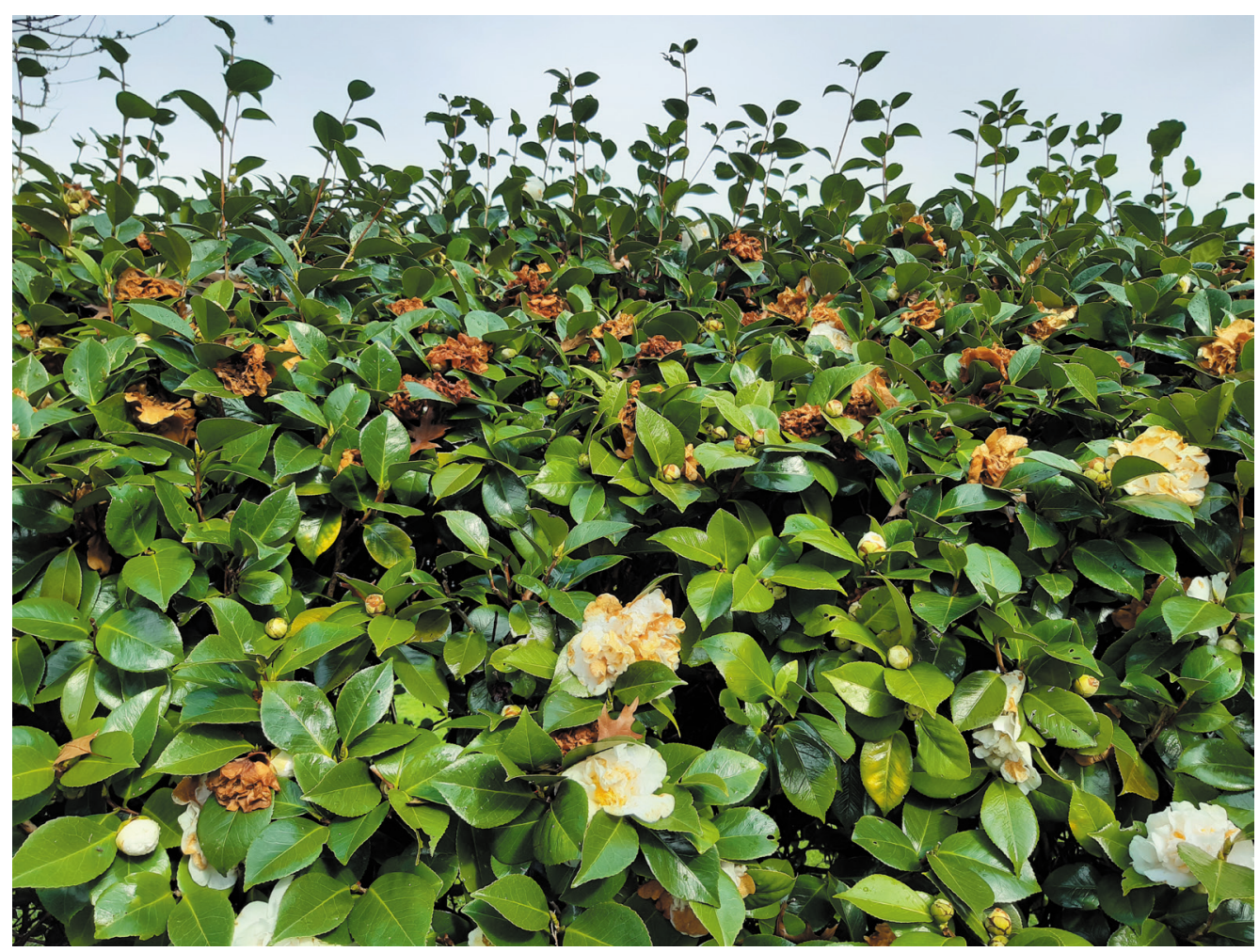

Fig. 2 Brown flowers of Camellia japonica 'Kate Sheppard'. Photo: Rebecca Stanley. 


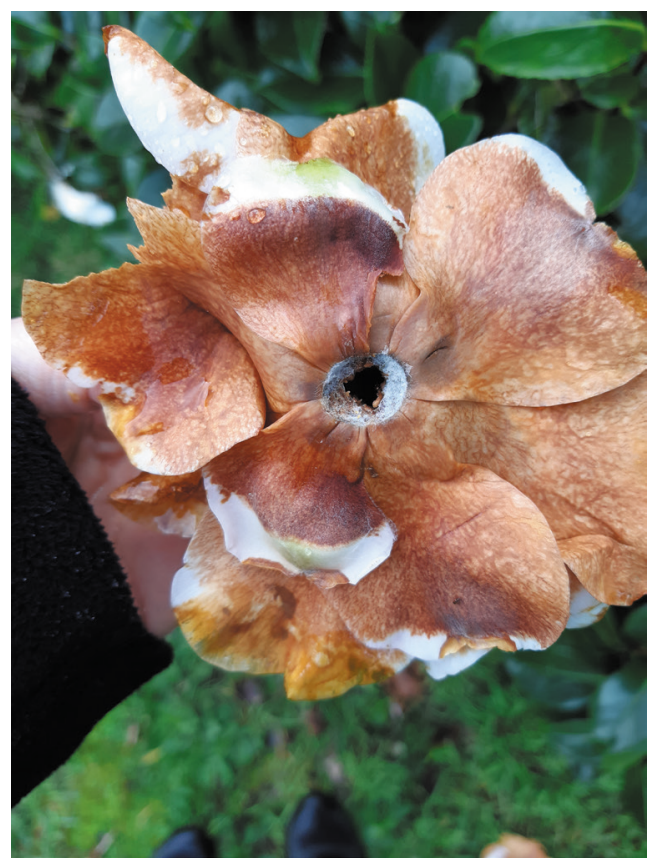

Fig. 3 Grey ring on mould around the base of a Camellia japonica 'Kate Sheppard' flower. Photo: Rebecca Stanley.

(Taylor \& Long, 2000). Since as far back as 1919, C. camelliae has been found in other countries, including Japan, France, England, Spain, Italy, Germany, Switzerland, Portugal and the United States, but it did not become widespread until the 1970s (Taylor \& Long, 2000). In the late 1990 s, a survey undertaken throughout New Zealand found it to be widespread and likely to be dispersed by wind and the transfer of infected material (Taylor, 1999).

Ciborinia camelliae is a host-specialist pathogen that only affects plants in the genus Camellia. Apothecia develop on sclerotia in spring and are dispersed by wind. These ascospores infect camellia petals quickly, causing the flowers to fall from the plant onto the ground (Taylor \& Long, 2000). When the flowers are on the ground, the sclerotia develop on the petals and overwinter on the rotting flowers (Taylor \&
Long, 2000) until environmental conditions are suitable for starting the cycle of infection again. This means the fungus does not require a second species to complete its reproductive cycle.

There are no strategies that prevent or control the annual reincursion of this fungus despite testing in various stages of the Ciborinia camelliae lifecycle with fungicides (Couselo et al., 2014). The disease affects larger-flowered, spring-blooming cultivars (japonica types) more frequently, partly because they flower later when the fungus is more active. The fungus is most active after a period of cool temperature followed by warming temperatures and moisture (American Camellia Society, 2020). However, spores can remain dormant for approximately four years until favourable conditions are met (Taylor, 2004). The disease is also more visible on larger and palerflowered blooms.

Auckland Botanic Gardens (ABG) has had a significant collection of Camellia species and cultivars - a total of 521 varieties since the gardens opened in 1982 (Fig. 4). The collection was planted in 1985 with the help of the late Neville Haydon, the former owner of Camellia Haven nursery. There are few studies of camellia petal blight's impact in gardens; however, some work has gone into testing a range of 39 Camellia for petal blight resistance. These range from C. pitardii $\times$ japonica 'Nicky Crisp' (Fig. 5), which has no resistance to the fungus, to C. lutchuensis (Fig. 6), which has high resistance (Denton-Giles et al., 2013). A breeding programme at ABG applies the knowledge from genetic work carried out by Denton-Giles et al. (2013) to select larger-flowered cultivars resistant to petal blight. The aim of this breeding programme is to develop a beautiful, 


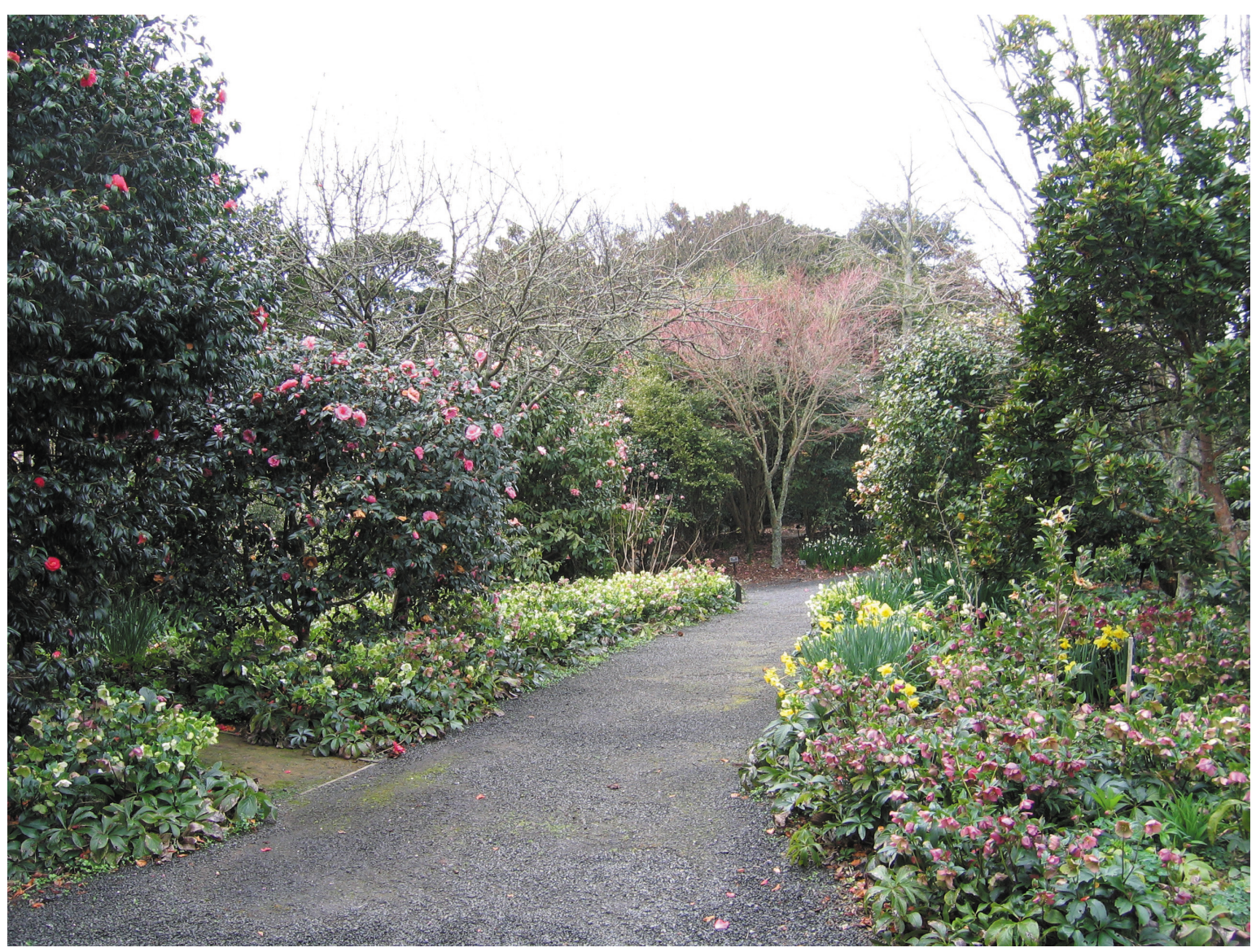

Fig. 4 Auckland Botanic Gardens' Camellia Garden contains a range of species and cultivars. Photo: Emma Bodley.

large-flowered camellia with an attractive habit, suitable for a small home garden and that is petal blight resistant. The breeding programme began in 2014 and it will take several more years before results are seen. Regular updates about the breeding programmes are published on the ABG website (Auckland Botanic Gardens, 2020). Denton-Giles et al. (2013) used the ABG Camellia collection to sample species; it was an easy task to sample 43 species in the Camellia Garden where all species camellias are planted together across two beds, a task that in the wild would be more costly and time-consuming.

\section{Methods}

Staff and students surveyed the ABG Camellia collection (1769794.95, 5902489.15 NZTM) during the flowering season in 2006, 2007, 2010, 2016, 2017 and 2018. A list of camellias in the collection was exported from the Plant Management Database with their locations to make surveying easier. A total of 363 camellias were surveyed between April and October over the six flowering seasons. For each camellia the presence or absence of petal blight was recorded. Five to ten flowers were assessed. If there was any doubt regarding a camellia's susceptibility to 


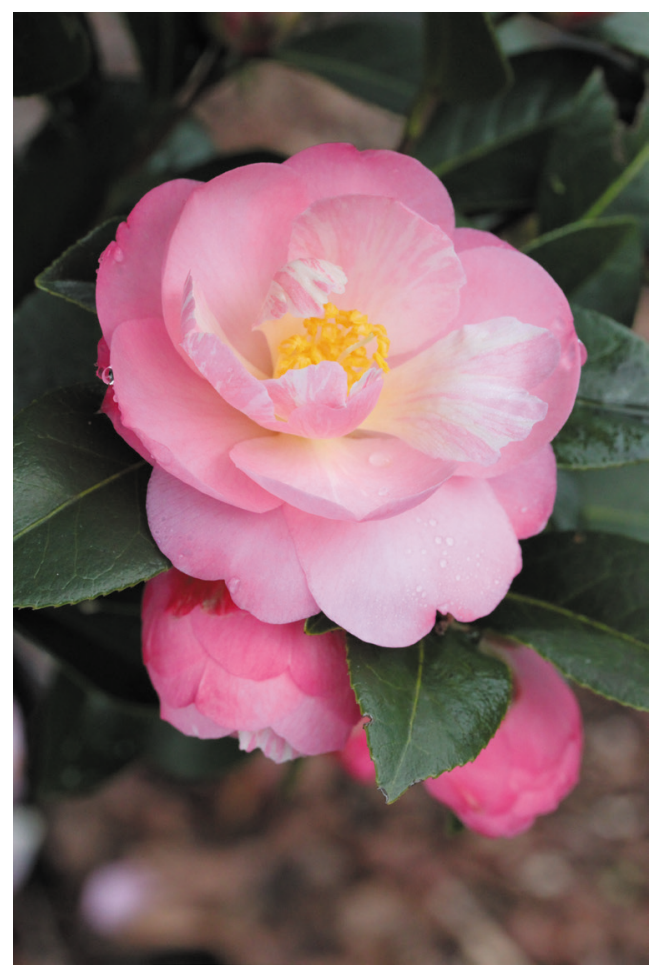

Fig. 5 Camellia pitardii $\times$ japonica 'Nicky Crisp' is susceptible to petal blight. Photo: Jack Hobbs.

petal blight during the initial assessment, cultivars were reassessed later in the flowering season. Detailed phenology records were not recorded during these surveys. Notes on phenology were, however, made on specific cultivars to help inform when to recheck flowers for petal blight.

\section{Results}

From the multiple surveys, we compiled a list of 245 cultivars which are susceptible to petal blight (Table 1) and a list of 68 cultivars on which we have not yet observed natural petal blight infections (Tables $2 \& 4$ ). Of these 68 that show no visual symptoms of petal blight, 48 are autumn-flowering cultivars, and 18 begin flowering in winter or spring. Six species are susceptible to petal blight, and 32 species show no visual symptoms of the disease. Many of the cultivars that have no visual symptoms of petal blight have a resistant species in their parentage, such as Camellia lutchuensis (Fig. 6). Although only the presence or absence of petal blight was recorded, we noted that some cultivars displayed symptoms differently. Dark-coloured flowers are less impacted aesthetically by petal blight because the brown petals are not as obvious in comparison to lighter flower colours. White and light pink flowers are aesthetically less attractive when infected with petal blight because the contrast between the light petals and the brown rot is greater.

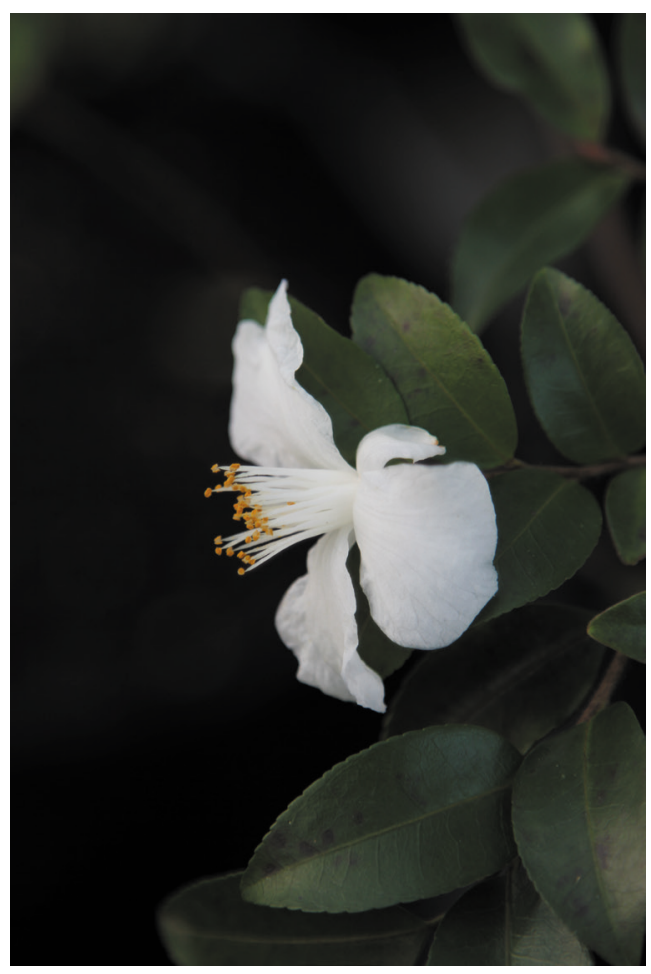

Fig. 6 Petal blight resistant Camellia lutchuensis. Photo: Jack Hobbs. 
Table 1 List of Camellia cultivars (total = 245) that are susceptible to petal blight in the Camellia Garden at ABG.

\begin{tabular}{|c|c|}
\hline Camellia 'Apple Blossom Sun' & Camellia japonica 'Dorothy James' \\
\hline Camellia'Bett's Supreme' & Camellia japonica 'Dr Lilyan Hanchey' \\
\hline Camellia 'Conquettii' & Camellia japonica'Dryade' \\
\hline Camellia 'Court Jester' & Camellia japonica 'Easter Morn' \\
\hline Camellia 'Lin Ryu' & Camellia japonica 'Ecclefield' \\
\hline Camellia'Mudan Cha' & Camellia japonica'Ed Combatalade' \\
\hline Camellia complex granthamiana hybrid 'Parisienne' & Camellia japonica'Elaine's Betty' \\
\hline Camellia complex japonica hybrid 'Superscent' & Camellia japonica 'Eleanor Martin Supreme' \\
\hline Camellia complex reticulata hybrid 'Anzac' & Camellia japonica'Elegans' \\
\hline Camellia complex saluenensis hybrid 'Fairy Wand' & Camellia japonica'Elegans Champagne' \\
\hline Camellia cuspidata hybrid 'Spring Festival' & Camellia japonica'Elegans Splendor' \\
\hline Camellia cuspidata $\times$ saluenensis 'Bellbird' & Camellia japonica'Elegans Supreme' \\
\hline Camellia cuspidata $\times$ saluenensis 'Cornish Snow' & Camellia japonica 'Elsie Ruth Marshall' \\
\hline Camellia japonica 'Alfred Upson' & Camellia japonica'Firedance' \\
\hline Camellia japonica 'Amazing Graces' & Camellia japonica'Fragrant Boutonniere' \\
\hline Camellia japonica 'Angel' & Camellia japonica 'Gayle Walden' \\
\hline Camellia japonica'Aquarius' & Camellia japonica 'Grace Albritton' \\
\hline Camellia japonica 'Ave Maria' & Camellia japonica 'Grand Slam' \\
\hline Camellia japonica 'Baby Pearl' & Camellia japonica 'Gwenneth Morey' \\
\hline Camellia japonica 'Berenice Boddy' & Camellia japonica'Hakuhan-kujaku' \\
\hline Camellia japonica'Betty Sheffield Supreme' & Camellia japonica'Hanafuki' \\
\hline Camellia japonica'Black Pearl' & Camellia japonica 'Harriet Bisbee' \\
\hline Camellia japonica'Black Tie' & Camellia japonica'Harry Cave' \\
\hline Camellia japonica 'Black Velvet' & Camellia japonica 'Hishikaraito' \\
\hline Camellia japonica'Blaze of Glory' & Camellia japonica 'Holly Bright' \\
\hline Camellia japonica'Bonbon Red' & Camellia japonica'Honeyglow' \\
\hline Camellia japonica 'Can Can' & Camellia japonica'Hopkin's Pink' \\
\hline Camellia japonica 'Canterbury' & Camellia japonica'In the Pink' \\
\hline Camellia japonica 'Carter's Sunburst Blush' & Camellia japonica'James Lockington' \\
\hline Camellia japonica 'China Doll' & Camellia japonica'Jerry Donnan' \\
\hline Camellia japonica 'Clarise Carleton' & Camellia japonica'Kate Sheppard' \\
\hline Camellia japonica 'C. M. Hovey' & Camellia japonica 'Katie' \\
\hline Camellia japonica 'C. M. Wilson' & Camellia japonica'Kick Off' \\
\hline Camellia japonica 'Commander Mulroy' & Camellia japonica'Kramer's Beauty' \\
\hline Camellia japonica 'Coronation' & Camellia japonica'K. Sawada' \\
\hline Camellia japonica 'Dark of the Moon' & Camellia japonica'Lady Clare' \\
\hline Camellia japonica 'Debutante' & Camellia japonica 'Lady Loch' \\
\hline Camellia japonica 'Desire' & Camellia japonica'Lady Macon' \\
\hline Camellia japonica 'Diddy's Pink Organdie' & Camellia japonica'Lady Vansittart Pink' \\
\hline Camellia japonica 'Dolly Dyer' & Camellia japonica'Laurie Bray' \\
\hline Camellia japonica 'Dona Herzilia de Freitas Magalhaes' & Camellia japonica'Leah Homeyer' \\
\hline
\end{tabular}




\begin{tabular}{|c|c|}
\hline Camellia japonica 'Lemon Drop' & Camellia japonica 'Prince Frederick William' \\
\hline Camellia japonica 'Little Bit Red' & Camellia japonica 'Queen Diana' \\
\hline Camellia japonica'Little Bit Striped' & Camellia japonica'R. L. Wheeler' \\
\hline Camellia japonica 'Little Ginger' & Camellia japonica 'Roger Hall' \\
\hline Camellia japonica 'Little Man' & Camellia japonica 'Royal Velvet' \\
\hline Camellia japonica'Little Michael' & Camellia japonica'Rudolph' \\
\hline Camellia japonica 'Look Away' & Camellia japonica 'Ruffian' \\
\hline Camellia japonica 'Lovelight' & Camellia japonica 'San Dimas' \\
\hline Camellia japonica 'Lover Boy' & Camellia japonica'Sanpei-tsukbaki' \\
\hline Camellia japonica'L. T. Dees' & Camellia japonica'Scentsation' \\
\hline Camellia japonica 'Lulu Belle' & Camellia japonica 'Show Time' \\
\hline Camellia japonica 'Mahogany Glow' & Camellia japonica 'Silver Anniversary' \\
\hline Camellia japonica 'Man Size' & Camellia japonica 'Silver Chalice' \\
\hline Camellia japonica'Manuroa Road' & Camellia japonica 'Silver Tower' \\
\hline Camellia japonica'Margaret Davis' & Camellia japonica 'Silver Waves' \\
\hline Camellia japonica'Mark Alan' & Camellia japonica 'Something Beautiful' \\
\hline Camellia japonica 'Maroon and Gold' & Camellia japonica 'Stacy Susan' \\
\hline Camellia japonica'Mary J. Wheeler' & Camellia japonica'Sundae' \\
\hline Camellia japonica'Mary Paige' & Camellia japonica 'Sunset Oaks' \\
\hline Camellia japonica'Maui' & Camellia japonica 'Swan Lake' \\
\hline Camellia japonica 'Michael's Joy' & Camellia japonica'Taba Meibi' \\
\hline Camellia japonica'Midnight' & Camellia japonica'Takanini' \\
\hline Camellia japonica 'Midnight Variegated' & Camellia japonica 'The Czar' \\
\hline Camellia japonica 'Mini Pink' & Camellia japonica 'Thomasville Beauty' \\
\hline Camellia japonica'Moonlight Bay' & Camellia japonica'Tiffany' \\
\hline Camellia japonica 'Moshio' & Camellia japonica'Tinsie' \\
\hline Camellia japonica'Mrs D. W. Davis' & Camellia japonica 'Tom Thumb' \\
\hline Camellia japonica'Mrs D. W. Davis Descanso' & Camellia japonica'Tootsie' \\
\hline Camellia japonica'Mrs Swan' & Camellia japonica 'Twilight' \\
\hline Camellia japonica'Nanbanko' & Camellia japonica'Ville De Nantes' \\
\hline Camellia japonica'Nick Carter' & Camellia japonica'Violet Bouquet' \\
\hline Camellia japonica 'Nuccio's Cameo' & Camellia japonica 'Virginia Franco Rosea' \\
\hline Camellia japonica'Nuccio's Carousel' & Camellia japonica'Volcano' \\
\hline Camellia japonica 'Nuccio's Gem' & Camellia japonica'Volunteer' \\
\hline Camellia japonica'Nuccio's Jewel' & Camellia japonica 'Wendy' \\
\hline Camellia japonica 'Nuccio's Pink Lace' & Camellia japonica 'Wilamina' \\
\hline Camellia japonica'Onetia Holland' & Camellia japonica'Wildfire' \\
\hline Camellia japonica 'Pilida' & Camellia japonica 'William Jackson' \\
\hline Camellia japonica 'Pink Smoke' & Camellia japonica'Yukimiguruma' \\
\hline Camellia japonica'Pirate's Gold' & Camellia japonica $\times$ fraterna 'Christmas Daffodil' \\
\hline Camellia japonica'Pride of California' & Camellia japonica $\times$ fraterna'Paddy's Perfumed' \\
\hline Camellia japonica 'Prima Ballerina' & Camellia japonica $\times$ lutchuensis 'Cinnamon Cindy' \\
\hline
\end{tabular}


Table 1 List of Camellia cultivars (total = 245) that are susceptible to petal blight in the Camellia Garden at ABG (cont.).

\begin{tabular}{|c|c|}
\hline Camellia japonica $\times$ reticulata 'Fire Chief Variegated' & Camellia saluenensis hybrid 'Ole' \\
\hline Camellia japonica $\times$ reticulata 'Harold L. Paige' & Camellia saluenensis $\times$ reticulata 'Barbara Clark' \\
\hline Camellia pitardii 'Snippet' & Camellia saluenensis $\times$ reticulata 'Brian' \\
\hline Camellia pitardii hybrid 'Annette Carol' & Camellia saluenensis $\times$ reticulata 'Fair Lass' \\
\hline Camellia pitardii hybrid 'Autumn Herald' & Camellia saluenensis $\times$ reticulata 'Francie $L^{\prime}$ \\
\hline Camellia pitardii hybrid 'Festival of Lights' & Camellia saluenensis $\times$ reticulata 'Innovation' \\
\hline Camellia pitardii hybrid 'Gay Pixie' & Camellia sasanqua 'Moon Moth' \\
\hline Camellia pitardii hybrid 'Hilda Jaggs' & Camellia williamsii $\times$ reticulata 'Blissful Dawn' \\
\hline Camellia pitardii hybrid 'Nonie Haydon' & Camellia × pitardii hybrid 'Adorable' \\
\hline Camellia pitardii hybrid 'Pearly Cascade' & Camellia $\times$ williamsii 'Angel Wings' \\
\hline Camellia pitardii hybrid 'Pink Cameo' & Camellia $\times$ williamsii 'Anticipation' \\
\hline Camellia pitardii hybrid 'Pink Ruffles' & Camellia $\times$ williamsii 'Ballet Queen' \\
\hline Camellia pitardii hybrid 'Purple Fire' & Camellia $\times$ williamsii 'Buttons 'n Bows' \\
\hline Camellia pitardii hybrid 'Rhonda Elizabeth' & Camellia $\times$ williamsii 'Daintiness' \\
\hline Camellia pitardii hybrid 'Sprite' & Camellia $\times$ williamsii 'Debbie' \\
\hline Camellia pitardii hybrid 'Vonnie Cave' & Camellia × williamsii 'Delores Edwards' \\
\hline Camellia pitardii $\times$ japonica 'Contemplation' & Camellia $\times$ williamsii 'Donation' \\
\hline Camellia pitardii × japonica 'Dr Colin Crisp' & Camellia $\times$ williamsii 'Dreamboat' \\
\hline Camellia pitardii × japonica 'Garnet Gleam' & Camellia × williamsii ‘Dresden China' \\
\hline Camellia pitardii × japonica'Jury's Pearl' & Camellia $\times$ williamsii 'E. G. Waterhouse’ \\
\hline Camellia pitardii × japonica 'Nicky Crisp' & Camellia $\times$ williamsii 'Elsie Jury' \\
\hline Camellia reticulata 'Chrysanthemum Petal' & Camellia $\times$ williamsii 'Hari Withers' \\
\hline Camellia reticulata'Moutancha' & Camellia × williamsii 'Joe Nuccio' \\
\hline Camellia reticulata 'Tom Durrant' & Camellia $\times$ williamsii 'Jury's Sunglow' \\
\hline Camellia reticulata 'Tongzimian' & Camellia $\times$ williamsii 'Jury's Yellow' \\
\hline Camellia reticulata $\times$ granthamiana 'Lois Shinault' & Camellia × williamsii 'Les Jury' \\
\hline Camellia reticulata $\times$ japonica 'Beth Dean' & Camellia $\times$ williamsii 'Little Lavender' \\
\hline Camellia reticulata $\times$ japonica 'Den Burton' & Camellia × williamsii 'Marjory Ramsey' \\
\hline Camellia reticulata $\times$ japonica 'Dr Clifford Parks' & Camellia × williamsii 'Mary Phoebe Taylor' \\
\hline Camellia reticulata $\times$ japonica 'Lasca Beauty' & Camellia $\times$ williamsii 'Mirage' \\
\hline Camellia reticulata $\times$ japonica 'Lovely Lady' & Camellia $\times$ williamsii 'Moonsong' \\
\hline Camellia reticulata $\times$ japonica 'Red Crystal' & Camellia × williamsii ‘Our Betty Variegated' \\
\hline Camellia reticulata $\times$ japonica 'Ruta Hagman' & Camellia × williamsii 'Rendezvous' \\
\hline Camellia reticulata $\times$ japonica 'Valentine's Day' & Camellia $\times$ williamsii 'Rose Bouquet' \\
\hline Camellia reticulata $\times$ saluenensis 'Massee Lane' & Camellia $\times$ williamsii 'Rose Holland' \\
\hline Camellia reticulata $\times$ sasanqua 'Gael's Dream' & Camellia × williamsii 'Softly' \\
\hline Camellia rusticana'Botanyuki' & Camellia $\times$ williamsii 'Sun Song' \\
\hline Camellia rusticana 'Himatsuri' & Camellia $\times$ williamsii 'Twinkle Star' \\
\hline Camellia rusticana $\times$ lutchuensis 'Fragrant Joy' & Camellia $(\times$ williamsii) $\times$ japonica 'Night Rider' \\
\hline Camellia saluenensis hybrid 'Itty Bit' & \\
\hline
\end{tabular}


Table 2 List of Camellia cultivars (total = 48) in the Camellia Garden at ABG that have no observed petal blight infections and are autumn-flowering.

\begin{tabular}{|c|c|}
\hline Camellia 'Bett's Surprise' & Camellia sasanqua'Paradise Belinda' \\
\hline Camellia chekiangoleosa 'Earlybright' & Camellia sasanqua'Paradise Gillian' \\
\hline Camellia complex rosiflora hybrid 'Wirlinga Gem' & Camellia sasanqua 'Paradise Hilda' \\
\hline Camellia complex tsaii hybrid 'Wirlinga Bride' (PBR) & Camellia sasanqua 'Paradise Joan' \\
\hline Camellia japonica 'Cho-cho-san' & Camellia sasanqua 'Paradise Little Liane' \\
\hline Camellia japonica'Lily Pons' & Camellia sasanqua 'Paradise Pearl' \\
\hline Camellia japonica 'Luana's Angel' & Camellia sasanqua 'Paradise Vanessa' \\
\hline Camellia japonica'Steve Blount' & Camellia sasanqua'Plantation Pink' \\
\hline Camellia japonica'Tama-no-ura' & Camellia sasanqua'Rosette Blush' \\
\hline Camellia japonica $\times$ lutchuensis 'Cinnamon Scentsation' & Camellia sasanqua'Setsugekka' \\
\hline Camellia japonica $\times$ lutchuensis 'Koto-no-kaori' & Camellia sasanqua'Taishuhai' \\
\hline Camellia kissii hybrid 'Buttermint' & Camellia sasanqua 'Weeping Maiden' \\
\hline Camellia lutchuensis hybrid 'Fairy Blush' & Camellia sasanqua $\times$ fraterna'Yoimachi' \\
\hline Camellia oleifera'Jaune' & Camellia williamsii × lutchuensis 'Sugar and Spice' \\
\hline Camellia oleifera $\times(\times$ hiemalis 'Snow Flurry') & Camellia $\times$ hiemalis 'Bonanza' \\
\hline Camellia sasanqua 'Double Rainbow' & Camellia $\times$ hiemalis 'Chansonette' \\
\hline Camellia sasanqua'Dwarf Shishi' & Camellia $\times$ hiemalis 'Dazzler' \\
\hline Camellia sasanqua 'Early Pearly' & Camellia $\times$ hiemalis 'Kanjiro' \\
\hline Camellia sasanqua 'Exquisite' & Camellia $\times$ hiemalis 'Shishigashira' \\
\hline Camellia sasanqua 'Gay Border' & Camellia $\times$ hiemalis 'Winsome' \\
\hline Camellia sasanqua'Jennifer Susan' & Camellia $\times$ vernalis 'Yuletide' \\
\hline Camellia sasanqua 'Marie Steiner' & Camellia $\times$ williamsii 'Angel Wings' \\
\hline Camellia sasanqua 'Midnight Lover' & Camellia $\times$ williamsii 'Mini Mint' \\
\hline Camellia sasanqua'Mine-no-yuki' & Camellia $\times$ williamsii 'Red Dragon' \\
\hline
\end{tabular}


Table 3 List of Camellia species infected (petal blight present) or not visibly affected (petal blight absent) in the field at ABG.

\begin{tabular}{|c|c|c|c|}
\hline Name & $\begin{array}{l}\text { Petal blight } \\
\text { present }\end{array}$ & $\begin{array}{l}\text { Petal blight } \\
\text { absent }\end{array}$ & $\begin{array}{l}\text { Previous research that } \\
\text { supports our findings }\end{array}$ \\
\hline Camellia bailinschanica & 1 & & \\
\hline Camellia caudata & & 1 & Denton-Giles, 2013 \\
\hline Camellia chekiangoleosa & & 1 & \\
\hline Camellia crassipes & & 1 & Denton-Giles, 2013 \\
\hline Camellia cuspidata & & 1 & Taylor, 2004 \\
\hline Camellia euryoides & & 1 & \\
\hline Camellia flava & & 1 & \\
\hline Camellia fluviatilis & & 1 & \\
\hline Camellia forrestii & & 1 & Taylor, 2004 \\
\hline Camellia fraterna & & 1 & Taylor, 2004; Denton-Giles, 2013 \\
\hline Camellia granthamiana & & 1 & \\
\hline Camellia grijsii & & 1 & Taylor, 2004 \\
\hline Camellia handelii & & 1 & \\
\hline Camellia henryana & & 1 & Denton-Giles, 2013 \\
\hline Camellia huiliensis & & 1 & \\
\hline Camellia impressinervis & & 1 & \\
\hline Camellia irrawadiensis & & 1 & \\
\hline Camellia jinshajiangica & & 1 & \\
\hline Camellia lutchuensis & & 1 & Taylor, 2004; Denton-Giles, 2013 \\
\hline Camellia minutiflora & & 1 & \\
\hline Camellia miyagii & & 1 & Denton-Giles, 2013 \\
\hline Camellia nitidissima var. nitidissima & & 1 & \\
\hline Camellia oleifera & & 1 & \\
\hline Camellia pitardii var. pitardii & 1 & & \\
\hline Camellia polyodonta & 1 & & \\
\hline Camellia puniceiflora & & 1 & \\
\hline Camellia reticulata f. simplex & 1 & & \\
\hline Camellia rosiflora & & 1 & Taylor, 2004 \\
\hline Camellia salicifolia & & 1 & \\
\hline Camellia saluenensis & 1 & & \\
\hline Camellia sinensis & & 1 & \\
\hline Camellia sinensis var. sinensis f. rosea & & 1 & \\
\hline Camellia subintegra & 1 & & \\
\hline Camellia transarisanensis & & 1 & Denton-Giles, 2013 \\
\hline Camellia transnokoensis & & 1 & Taylor, 2004; Denton-Giles, 2013 \\
\hline Camellia trichocarpa & & 1 & Taylor, 2004 \\
\hline Camellia trichoclada & & 1 & \\
\hline Camellia tsaii & & 1 & Taylor, 2004 \\
\hline
\end{tabular}


Table 4 Winter- or spring-flowering camellias (total $=18$ ) with no observed petal blight infections at ABG.

\begin{tabular}{|l|l|}
\hline Camellia complex saluenensis hybrid 'Gay Baby' & Camellia japonica 'Yuri-tsubaki' \\
\hline Camellia fraterna $\times$ tsaii 'Ariel's Song' & Camellia japonica $\times$ fraterna 'Tiny Princess' \\
\hline Camellia japonica 'Bob's Tinsie' & Camellia japonica $\times$ lutchuensis 'Sweet Emily Kate' \\
\hline Camellia japonica 'Demi-Tasse' & Camellia lutchuensis $\times$ japonica 'Scented Gem' \\
\hline Camellia japonica 'Kumagai Nagoya' & Camellia rosiflora $\times$ tsaii 'Baby Bear' \\
\hline Camellia japonica 'Nokogiriba-tsubaki' & Camellia rosiflora $\times$ tsaii 'Baby Brother' \\
\hline Camellia japonica 'Nuccio's Pearl' & Camellia rosiflora $\times$ tsaii 'Baby Willow' \\
\hline Camellia japonica 'Sir Victor Davies' & Camellia $\times$ williamsii 'Wynne Rayner' \\
\hline Camellia japonica 'Sweet Olive' & Camellia $\times$ williamsii 'Mimosa Jury' \\
\hline
\end{tabular}

\section{Conclusion}

There are at least ten cultivars where susceptibility is unclear from field observations because flowering times have not been recorded; these will be confirmed in subsequent years (Appendix). This requires not only observing the presence or absence of petal blight, but also recording phenology to gain an understanding of whether resistance or avoidance is the mechanism for the lack of petal blight. Although camellias are not commonly grown by gardeners, we have compiled a list of Camellia species and our observations of the impact on them of petal blight (Table 3).

Until there is more success in breeding for resistance to camellia petal blight, we recommend that gardeners choose autumnflowering (sasanqua type) cultivars (Table 2) to avoid the unsightly effects of the disease. However, there are some late-flowering camellias that display no visual symptoms of petal blight which could be a result of their parentage providing some resistance (Table 4). These Camellia cultivars flower before Ciborinia camelliae releases its spores but some also have a certain resistance thanks to their parentage.
We provide an extensive list of petal blight free camellias and are happy to share this information with gardens, nurseries and other breeders. At ABG, we have gradually removed plants that have petal blight and so when people visit our garden, they will see healthy, petal blight free plants which will provide them with inspiration for planting at home. At ABG, we display plants that grow well in Auckland conditions, are free from pests or diseases and have spectacular flowering. We hope that this will help gardeners make choices about which cultivars to avoid planting and, in addition, will inform the ABG cultivar breeding programme with regard to the selection of resistant parents.

\section{Acknowledgements}

We want to thank the Camellia Society Auckland Branch for supporting Jess Ryder, Keely Paler and Matthew Savage in surveying.

\section{References}

AMERICAN CAMELLIA SOCIETY (2020). Camellia petal blight. Available online: https://www. americancamellias.com/care-culture-resources/ insects-and-diseases/camellia-petal-blight (accessed April 2020). 
AUCKLAND BOTANIC GARDENS (2020). Plant breeding programmes. Available online: http:// www.aucklandbotanicgardens.co.nz/science/ research/plant-breeding/ (accessed July 2020).

COUSELO, J.L., VELA, P., SALINERO, C. \&

MANSILLA, P. (2014). Limiting factors for the biological and chemical control of Camellia Flower Blight. In: Proceedings of the International Camellia Congress, Pontevedra, Spain. Deputación de Pontevedra, pp. 266-279.

DENTON-GILES, M., BRADSHAW, R. \& DIJKWEL, P. (2013). Ciborinia camelliae (Sclerotiniaceae) induces variable plant resistance responses in selected species of Camellia. Phytopathology, 103(7): 725-732.

TAYLOR, C.H. (1999). Studies of camellia flower blight (Ciborinia camelliae). Master's thesis. Massey University, New Zealand.

TAYLOR, C.H. (2004). Studies of Camellia flower blight (Ciborinia camelliae Kohn.). PhD dissertation. Massey University, New Zealand.

TAYLOR, C.H. \& LONG, P.G. (2000). Review of literature on camellia flower blight caused by Ciborinia camelliae. New Zealand Journal of Crop and Horticultural Science, 28: 123-138.

\section{Appendix}

The following is a list of camellias (total $=10$ ) in ABG's collection that have been recorded as having no petal blight. This is either because no record of flowering times has been documented to determine if they are autumn flowering or not, or because they are no longer held in the ABG collection. It cannot therefore be determined whether they are petal blight resistant or avoid petal blight by early flowering. This will be investigated in subsequent years.

\begin{tabular}{|l|}
\hline Camellia japonica 'Grape Soda' \\
\hline Camellia japonica 'Kikarako' \\
\hline Camellia japonica 'Prince Eugene Napoleon' \\
\hline Camellia japonica 'Red Ensign' \\
\hline Camellia pitardii $\times$ fraterna 'Alpen Glo' \\
\hline Camellia pitardii $\times$ japonica 'Persuasion' \\
\hline Camellia rosiflora 'Cascade' \\
\hline Camellia rosiflora hybrid 'Standard Bearer' \\
\hline Camellia rosiflora $\times$ fraterna 'Captured Enriches' \\
\hline Camellia $\times$ williamsii 'Water Lily' \\
\hline
\end{tabular}

